\title{
MicroRNA-198 contributes to lupus nephritis progression by inhibition of phosphatase and tensin homology deleted on chromosome ten expression
}

\author{
DANYU CUI* , DINGJI ZHU*, HAO REN, JINGLI LIN, WEINAN LAI, QIN HUANG, JINJUN ZHAO and MIN YANG \\ Department of Rheumatology and Immunology, Nanfang Hospital, \\ Southern Medical University, Guangzhou, Guangdong 510515, P.R. China
}

Received November 10, 2016; Accepted June 22, 2017

DOI: $10.3892 / \mathrm{mmr} .2017 .7527$

\begin{abstract}
A number of short noncoding microRNAs (miRs) have been demonstrated to be highly expressed in many kidney diseases such as renal cancer and lupus nephritis (LN); however, these results have not been extensively investigated. The aim of the present study was to investigate the expression and function of miR-198 in LN based on the previous studies. miR-198 expression level in systemic lupus erythematosus (SLE) patients was determined to determine its clinicopathological significance and effect on glomerular cell proliferation. It was demonstrated that higher expression of miR-198 was observed in patients with SLE, and was correlated with disease activity. Bioinformatics prediction and luciferase assays were used to demonstrate that miR-198 could directly bind to the phosphatase and tensin homology deleted on chromosome ten (PTEN) 3'-untranslated region. Furthermore, miR-198 overexpression reduced PTEN expression levels, while miR-198 silencing increased its expression at both the mRNA and protein level. Furthermore, there was a negative association between miR-198 and PTEN in the patients with active SLE. Thus, miR-198 may promote proliferation and contribute to SLE progression by targeting PTEN.
\end{abstract}

\section{Introduction}

Systemic lupus erythematosus (SLE) is a highly complex autoimmune disease (1), characterized by disorder of the generation of auto-antibodies to the cell nucleus components (2). Approximately 30-50\% of SLE patients are diagnosed with

Correspondence to: Dr Min Yang, Department of Rheumatology and Immunology, Nanfang Hospital, Southern Medical University, Northern 1838th Guangzhou Avenue, Guangzhou, Guangdong 510515, P.R. China

E-mail: minyangguangzhou@sina.com

\section{${ }^{*}$ Contributed equally}

Key words: microRNA-198, lupus nephritis, phosphatase and tensin homology deleted on chromosome ten, proliferation lupus nephritis (LN) (3), which is more prevalent in Asians and Africans compared with other races, and the 5-year survival rate of patients with severe $\mathrm{LN}$ is $<70-80 \%(4,5)$. $\mathrm{LN}$ is determined by immune system activation and the renal tissue response to inflammation with substantial morbidity and mortality. Although many advances have been made in its management, $10-30 \%$ of patients progress to end-stage renal disease. Therefore, finding novel non-invasive biomarkers at the early stages of SLE is of great interest.

MicroRNAs (miRNAs/miRs) are a group of highly conserved small non-coding, single-stranded RNA molecules comprised of $\sim 18-25$ bp in length (6). miRNAs regulate gene expression at the post-transcriptional level by binding to the 3'-untranslated region (UTR), which results in degrading or blocking translation of messenger RNA (mRNA) $(7,8)$. miRNAs have been implicated to serve important roles in several diseases due to their specificity, such as the pathogenesis of SLE $(9,10)$. Abnormal expression of miRNAs has been demonstrated in LN patients, such as in the blood serum, urine and renal tissue; however, these results have not been investigated extensively. Therefore, the present study aimed to investigate the expression and function of miR-198 in active LN, in order to further understand the molecular mechanisms. These results bring novel insight into understanding the mechanisms about the lupus disease pathogenesis.

\section{Materials and methods}

Patients. Adult SLE patients $(n=52)$ were enrolled between January 2012 and December 2014 from the Department of Rheumatology and Immunology, Nanfang Hospital. The present study was approved by the Ethics Committee of Southern Medical University (Guangzhou, China). Written informed consent was obtained from all patients. Among them, 30 patients with consecutive SLE and active nephritis underwent kidney biopsy, and 22 patients were diagnosed as inactive SLE. Sections of the kidneys were surgically resected due to kidney biopsy, and subsequently fixed in $4 \%$ formaldehyde at room temperature overnight for in situ hybridization at 3-4 $\mu \mathrm{m}$. The diagnosis was according to the American College of Rheumatology diagnostic criteria of SLE (11). The Systemic Lupus Erythematosus Disease Activity Index 
(SLEDAI) score was determined at the time of the blood draw; scores $<4$ were considered as inactive disease, and scores $\geq 4$ was categorized as active disease. Renal SLEDAI scores were determined as described (12). The mesangial cell proliferation was regarded as the main pathological change. A total of 10 paired renal tissue samples served as the control. Additionally, renal tissues were collected from 6 patients with Behcet's disease to compare SLE with other autoimmune disease. Patients with Behcet's disease were recruited from the Department of Rheumatology and Immunology, Nanfang Hospital. Sections of the kidneys were surgically resected due to pathological examination, which were subsequently fixed in $4 \%$ formaldehyde at room temperature overnight for in situ hybridization at 3-4 $\mu \mathrm{m}$.

Cell culture and transfection experiments. The MMC mouse mesangial cell line (cat. no. CRL-1927) and human embryonic kidney 293 (HEK-293) cells were obtained from the American Type Culture Collection (Manassas, VA, USA). The MMCs were cultured in Dulbecco's modified Eagle's medium (DMEM)/F12 (3:1; Gibco; Thermo Fisher Scientific, Inc., Waltham, MA, USA) supplemented with $10 \%$ fetal bovine serum (FBS; Thermo Fisher Scientific, Inc.). HEK-293 cells were maintained in DMEM supplemented with L-glutamine and 10\% FBS (Thermo Fisher Scientific, Inc.). Cells were transfected using the siPORTNeoFX agent with $50 \mathrm{nM}$ mirVana (Invitrogen; Thermo Fisher Scientific, Inc.). hsa-miR-198 mimic, hsa-miR-198 inhibitor or their respective negative controls [(NC); miR-NC, as-miR-NC] were used for transfection (Ambion; Thermo Fisher Scientific, Inc.). Three independent experiments were conducted. The transfected cells were collected for total RNA or protein isolation at $48 \mathrm{~h}$ post-transfection.

Reverse transcription-quantitative polymerase chain reaction $(R T-q P C R)$. Total RNA was extracted from mouse kidneys and culture cell lysates using TRIzol ${ }^{\circledR}$ (Invitrogen; Thermo Fisher Scientific, Inc.), according to the manufacturer's protocol. The concentration and purity of the RNA was confirmed using an Agilent 2100 system. RNA was reverse transcribed to cDNA using the High Capacity RNA-to-cDNA kit or a PrimeScript 1st strand cDNA Synthesis kit (Takara Biotechnology Co., Ltd., Dalian, China). qPCR was performed in 96-well plates on an ABI 7500 system using Taqman Gene Expression assay according to the manufacturer's protocol (Applied Biosystems; Thermo Fisher Scientific, Inc.). The data were normalized based on the expression of the endogenous control GAPDH (mRNA), and U6 small nuclear RNA was used for miRNA normalization. The primers used were as follows: GAPDH, forward 5'-GAGAAGTATGACAACAGC CTC-3' and reverse 5'-ATGGACTGTGGTCATGAGTC-3'; PTEN, forward 5'-AAGTCCAGAGCCATTTCCAT-3' and reverse 5'-CAAGTCTAAGTCGAATCCATCCT-3'; miR-198, forward 5'-TCATTGGTCCAGAGGGGAGATAG-3' and reverse 5'-GCAGGGTCCGAGGTATTC-3'; U6, forward 5'-CTCGCTTCGGCAGCACA-3', and reverse 5'-AACGCT TCACGAATTTGCGT-3'. The PCR amplification conditions were: $95^{\circ} \mathrm{C}$ for $10 \mathrm{~min}$, followed by 35 cycles of $95^{\circ} \mathrm{C}$ for $10 \mathrm{sec}, 60^{\circ} \mathrm{C}$ for $20 \mathrm{sec}$ and $72^{\circ} \mathrm{C}$ for $30 \mathrm{sec}$, then $4^{\circ} \mathrm{C}$ for $5 \mathrm{~min}$. $\mathrm{Cq}$ values were normalized to the endogenous control and presented as the fold-change relative to the control sample $(2 \Delta \Delta \mathrm{Cq})(13)$.

Bioinformatics analysis. TargetScan 6.2 (www.targetscan.org) was used to screen the potential targets of miR-198. PTEN was identified as one of the miR-198 candidate targets.

Luciferase assay. The phosphatase and tensin homology deleted on chromosome ten (PTEN) 3'-UTR luciferase reporter was cloned into the pGL3 luciferase reporter vector between the SpeI and HindIII sites. The mutant PTEN-3'UTR was generated as (TCTGGA to TCGGGA). The sequence was confirmed by DNA sequencing. MMCs and HEK-293 cells were cultured in a 24-well plate the day before transfection. Cells were co-transfected with the firefly luciferase-3-UTR (pGL3-PTEN or pGL3-PTEN mutant; $500 \mathrm{ng}$ ) and the pRL-TK vector (Promega Corporation, Madison, WI), along with the miR-198, miR-198 inhibitor or the control sequences. At $48 \mathrm{~h}$ after transfection, cell lysates were prepared and luciferase assays were performed using an HT microplate reader (BioTek China, Beijing, China). Luciferase activities were normalized to Renilla luciferase activity. All the experiments were repeated at least three times.

Cell proliferation assays. Cell proliferation was examined using a Cell Counting kit-8 (CCK-8; Beyotime Institute of Biotechnology, Haimen, China) at 0, 24, 48, 72, 96 and $120 \mathrm{~h}$ after inoculation by means of a cell proliferation assay according to the manufacturer's protocol. The optical density was measured at a wavelength of $450 \mathrm{~nm}$ using a microplate reader.

Western blotting. Mouse kidneys or HEK-293T cells were lysed using radioimmunoprecipitation assay buffer with complete protease inhibitor cocktail (Roche Diagnostics, Indianapolis, IN, USA). Proteins ( $30 \mu \mathrm{g})$ were separated by $10 \%$ SDS-PAGE and then transferred to a nitrocellulose membrane. After blocking with non-fat milk for $30 \mathrm{~min}$ at room temperature, the membrane was incubated overnight at $4^{\circ} \mathrm{C}$ with a PTEN primary antibody (MAB4037, 1:1,000 dilution, EMD Millipore, Billerica, MA, USA) or a mouse monoclonal anti- $\beta$-actin primary antibody (MABT825, 1:1,000 dilution, EMD Millipore). Horseradish peroxidase (HRP)-conjugated anti-rabbit (sc-2004, 1:3,000, Santa Cruz Biotechnology, Inc., Dallas, TX, USA) or anti-mouse IgG antibodies (sc-2005, 1:3,000, Santa Cruz Biotechnology, Inc.) was used as the secondary antibody. At last, the membranes were visualized with the ECL Western Blotting Detection System (GE Healthcare Life Sciences).

Statistical analysis. Statistical analysis was performed using SPSS version 17.0 (SPSS Inc., Chicago, IL, USA). The results are expressed as the mean \pm standard deviation. Comparison of parameters of the three groups (SLE, patients with Behcet's disease and normal controls) was carried out using one way analysis of variance/Kruskal-Wallis test. Multiple comparisons between the groups were performed using Student-Newman-Keuls method. The correlations between biomarker and the activity were calculated by Spearman's 
A

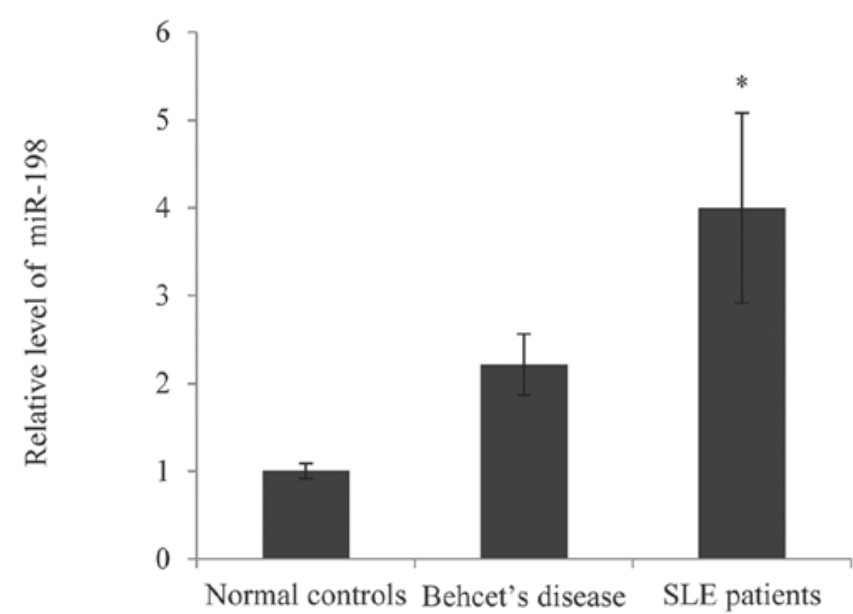

B
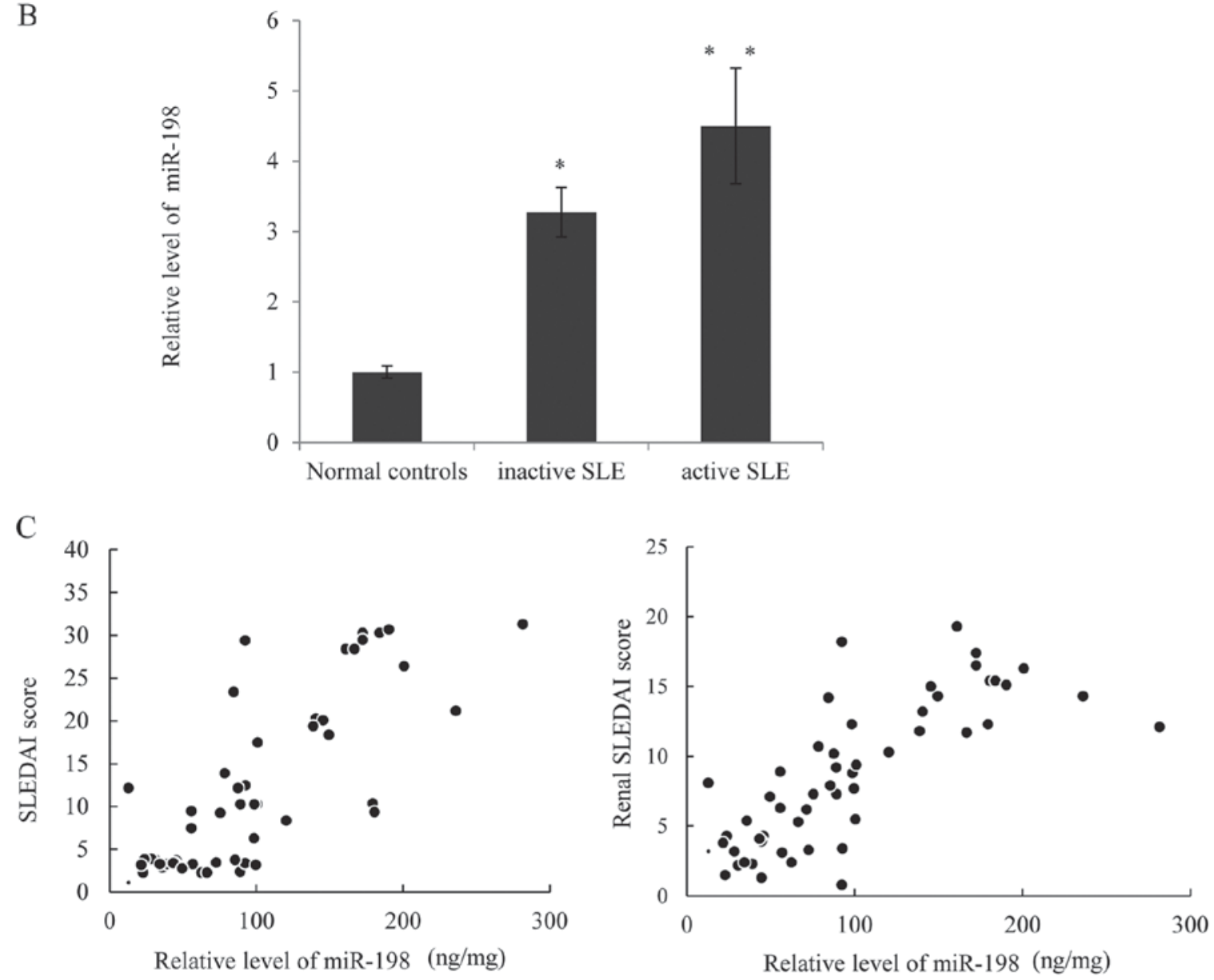

Figure 1. Higher expression of miR-198 is observed in patients with SLE, and is correlated with disease activity. (A) Expression of miR-198 was measured in 52 patients with SLE, 6 patients with Behcet's disease, and 10 paired control tissue samples. Data are presented as the mean \pm standard error. (B) Expression of miR-198 was measured in 30 patients with active SLE, 22 patients with inactive SLE, and 10 normal controls. Data are expressed as the mean \pm standard deviation. ${ }^{*} \mathrm{P}<0.05,{ }^{* *} \mathrm{P}<0.01$. (C) Correlation between miR-198 levels and the total SLEDAI score or the renal SLEDAI score was measured. SLEDAI, SLE Disease Activity Index; miR, microRnA; SLE, systemic lupus erythematosus.

rank correlation coefficient. $\mathrm{P}<0.05$ was considered to indicate a statistically significant difference.

\section{Results}

Higher expression of miR-198 is observed in patients with SLE, and is correlated with disease activity. RT-qPCR was used to examine miR-198 expression in the SLE patients and those with active nephritis. As presented in Fig. 1A, the expression of miR-198 was significantly higher in lupus patients compared with normal controls, and although the miR-198 expression in patients with Behcet's disease appeared higher than the controls, there was no statistical differences. Next, correlational analysis was performed to determine whether there was any correlation between the expression of miR-198 and clinical features. As presented 
A

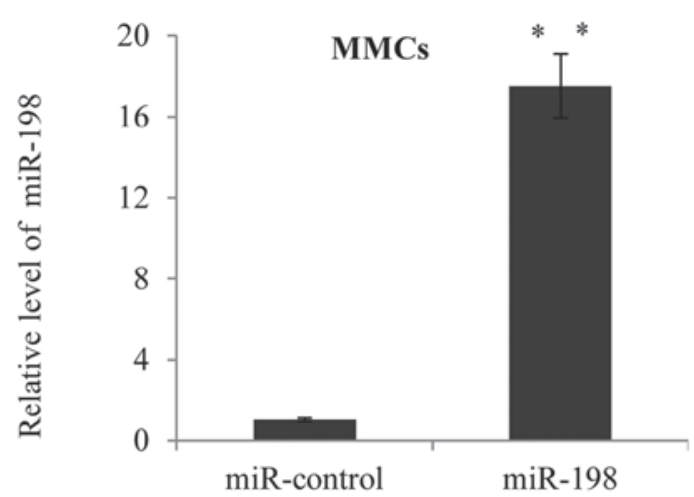

$\mathrm{B}$

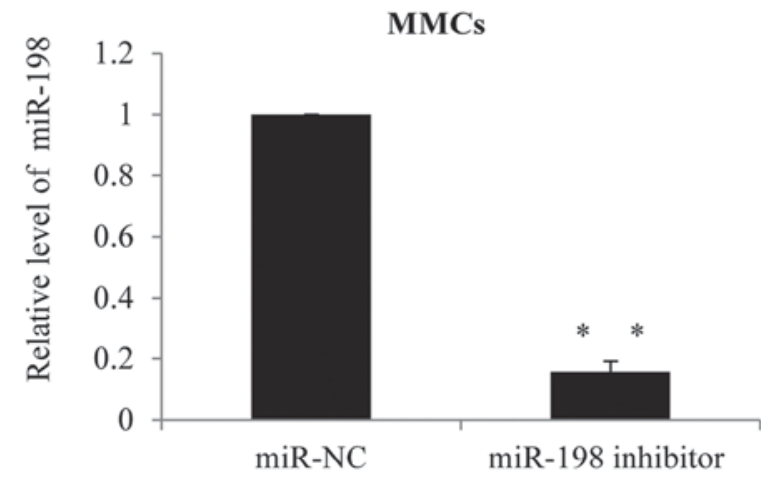

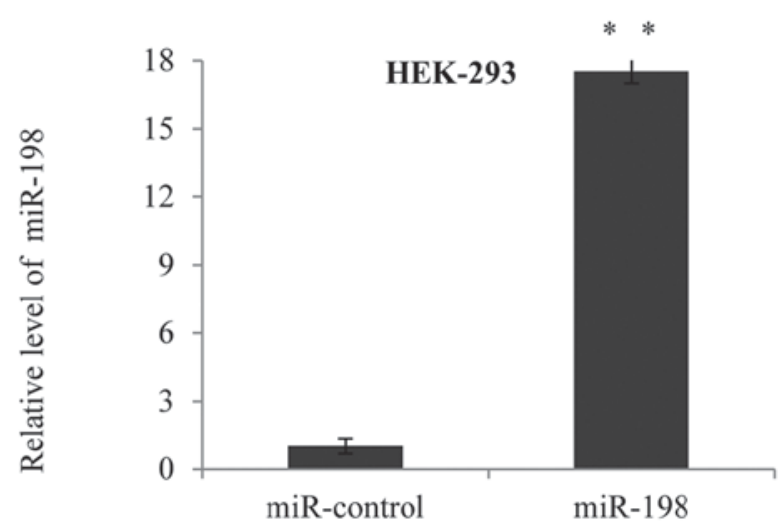

HEK-293

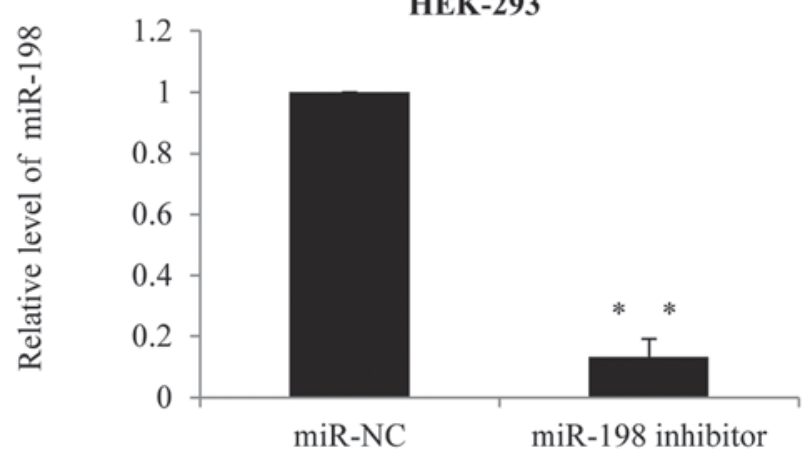

C

D
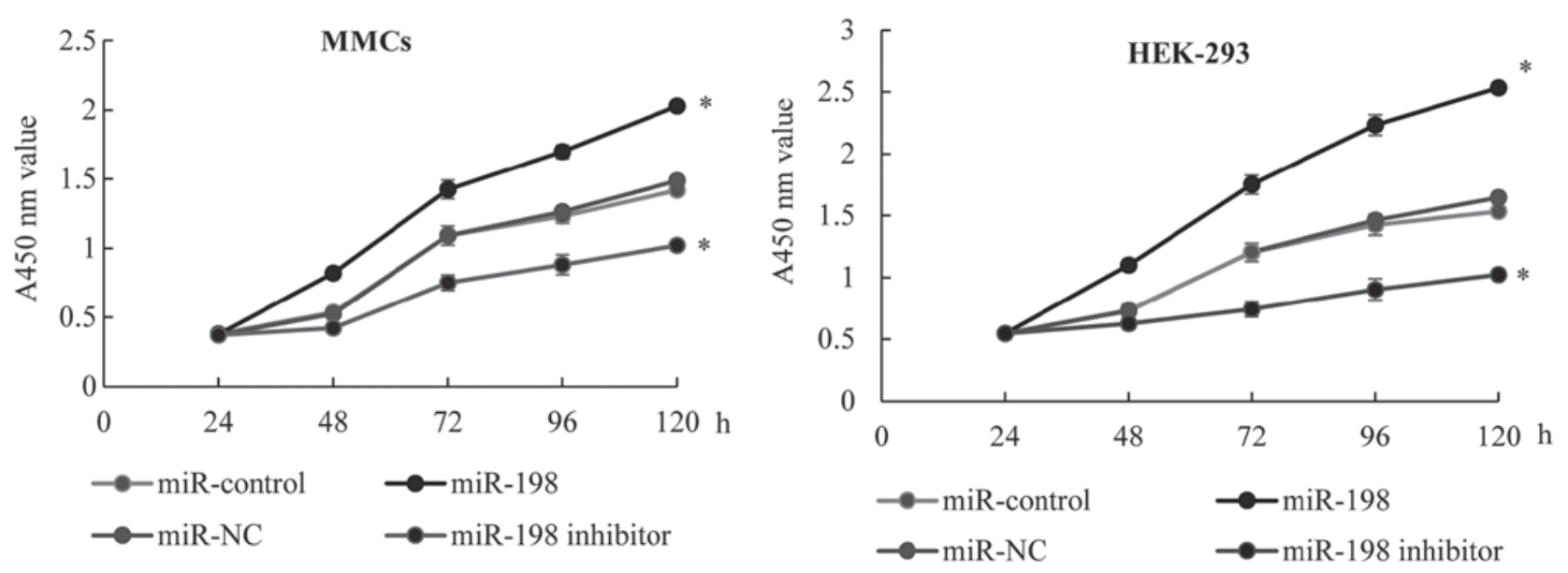

Figure 2. miR-198 promotes glomeruli cell growth and proliferation in LN. MMCs or HEK-293 cells were transfected with (A) miR-198 mimic or miR-control, and (B) miR-198 inhibitor or miR-NC, and assessed by reverse transcription-quantitative polymerase chain reaction. Cell-Counting kit-8 assay in (C) MMCs and (D) HEK-293 cells. Data are expressed as the mean \pm standard deviation of experiments conducted in triplicate. ${ }^{*} \mathrm{P}<0.05$, ${ }^{* *} \mathrm{P}<0.01$. LN, lupus nephritis; miR, microRNA; NC, negative control.

in Fig. 1B, the expression of miR-198 was higher in patients with active SLE compared with those with inactive SLE and the normal controls. Furthermore, a direct positive correlation was observed between miR-198 levels and the SLEDAI scores, as well as between the miR-198 levels and the renal SLEDAI scores (Fig. 1C). Thus, it was hypothesized that higher miR-198 expression positively correlates with SLE disease activity.

miR-198 promotes glomeruli cell growth and proliferation in $L N$. As high expression levels of miT-198 were observed in the LN group, it was hypothesized that miR-198 may correlate with glomeruli cell proliferation. To validate this hypothesis, a hsa miR-198 mimic, or hsa-miR-198 inhibitor or their respective controls were transfected into MMCs or HEK-293 cells. Successful overexpression (Fig. 2A) or downregulation (Fig. 2B) of miR-198 in the two cells was confirmed by RT-qPCR. Furthermore, a CCK-8 assay was performed in the MMCs (Fig. 2C) or HEK-293 (Fig. 2D) cells transfected with miR-control, miR-198, miR-NC or miR-198 inhibitor, and the growth ratio was observed. Overexpression of miR-198 could significantly increase the cell growth rates, while the knockdown of miR-198 remarkably inhibited the growth level. 
A

Relative position

Predicted consequential pairing of target

region(top) and miRNA(bottom)

hsa-miR-198

Position 541-565 of PTEN 3'UTR

\section{3'...CUUGGAUAGAGGGGGAGACCUGG....5' \\ IIIII I \\ 5 \\ AATTTTATGCTGGACTCTGGACCA 3,}

$\mathrm{B}$

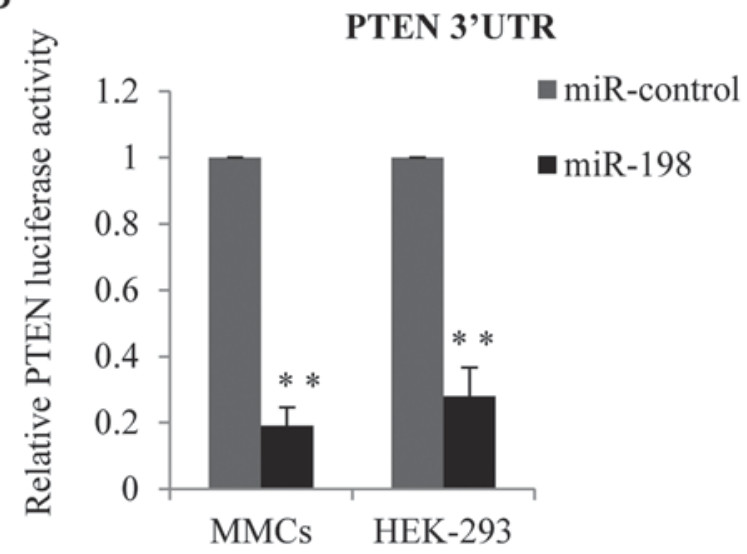

D

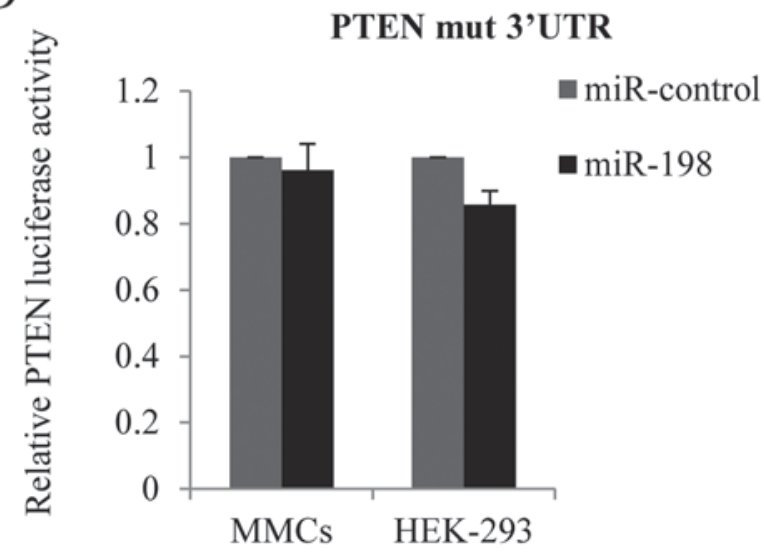

$\mathrm{C}$

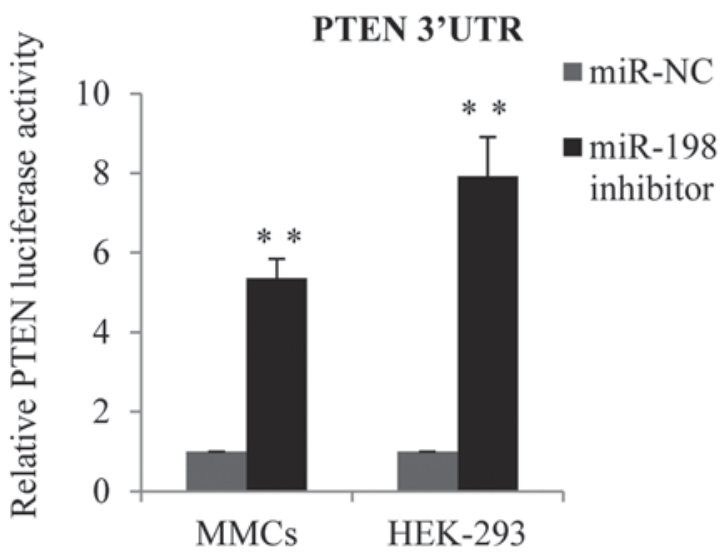

E

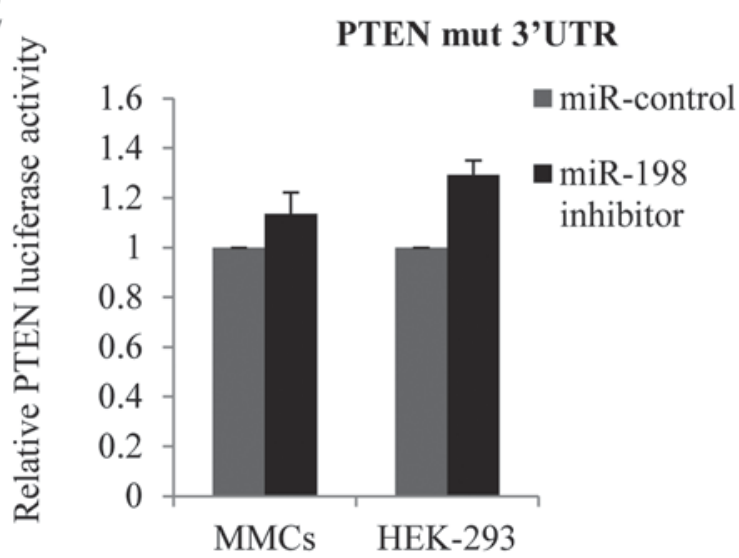

Figure 3. Validation of PTEN as a direct target of miR-198. (A) Schematic description of 3'-UTR of PTEN indicating the interactions between the PTEN 3'-UTR binding site and miR-198 using the TargetScan software. MMCs and HEK-293 cells were co-transfected with the luciferase gene of wild-type 3'-UTR PTEN and (B) miR-198 or miR-control, and (C) miR-198 inhibitor or miR-NC. MMCs and HEK-293 cells were co-transfected with the luciferase gene of mutant 3'-UTR PTEN and (D) miR-198 or miR-control, and (E) miR-198 inhibitor or miR-NC. Data are expressed as the mean \pm standard deviation. ${ }^{* *} \mathrm{P}<0.01$. PTEN, phosphatase and tensin homology deleted on chromosome ten; miR, microRNA; NC, negative control; UTR, untranslated region; mut, mutant.

Validation of PTEN as a direct target of miR-198. In order to elucidate the mechanism of miR-198 to stimulate cell proliferation, bioinformatics analysis was used to identify its target genes. TargetScan 6.2 (http://www.targetscan.org) was used to screen the potential target and PTEN was identified as one of the miR-198 candidate targets (Fig. 3A). To determine whether miR-198 can potentially bind to PTEN, a luciferase reporter assay was performed in the MMCs and HEK-293 cell lines. As presented in Fig. 3B, in cells transfected with miR-198 mimic, the luciferase activity of the PTEN-3'UTR was remarkably decreased compared with the control. Conversely, when cells were transfected with miR-198 inhibitor, the PTEN-3'UTR luciferase activity was obviously increased (Fig. 3C). However, when cells were transfected with mutant PTEN-3'UTR (TCTGGA to TCGGGA), the inhibition effect of miR-198 was almost abolished (Fig. 3D and E).

Forced expression of miR-198 inhibits PTEN expression. To investigate the possible mechanism underlying miR-198 in cell proliferation, the present study detected the function of miR-198 on the expression of PTEN using RT-qPCR. miR-198 reduced the expression of PTEN compared with the control cells (Fig. 4A) while the miR-198 inhibitor upregulated the expression of PTEN in MMCs and HEK-293 cells (Fig. 4B). Furthermore, western blot analysis was performed to analyze 
A

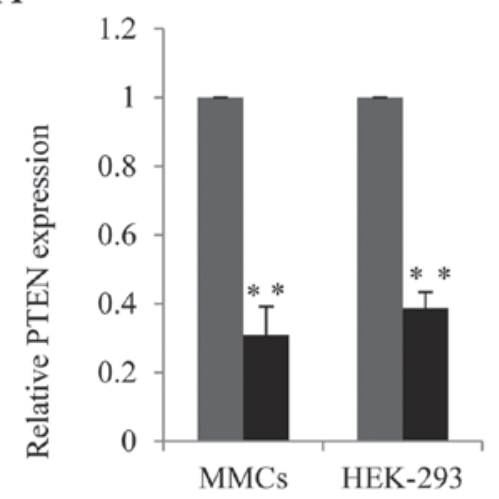

$\mathrm{C}$

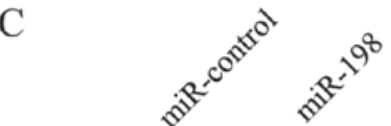

PTEN

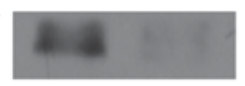

$\beta$-actin

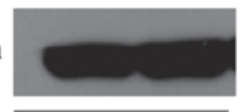

MMCs

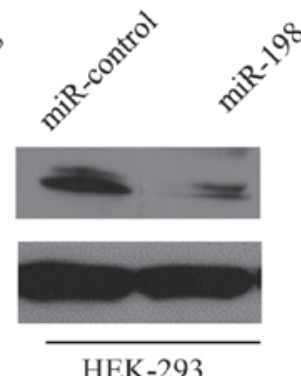

HEK-293
B

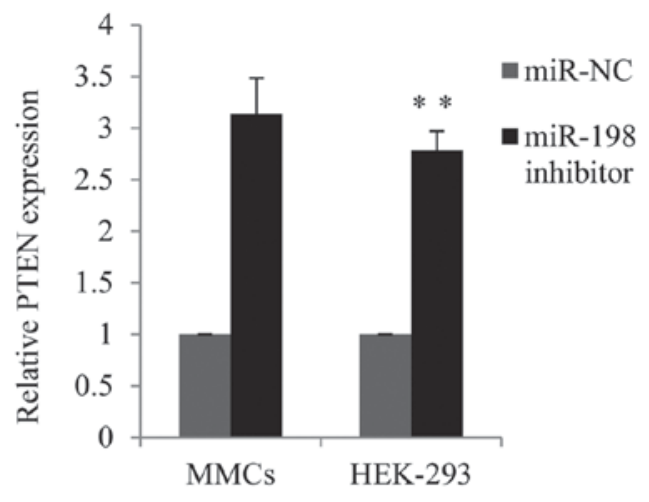

$\mathrm{D}$
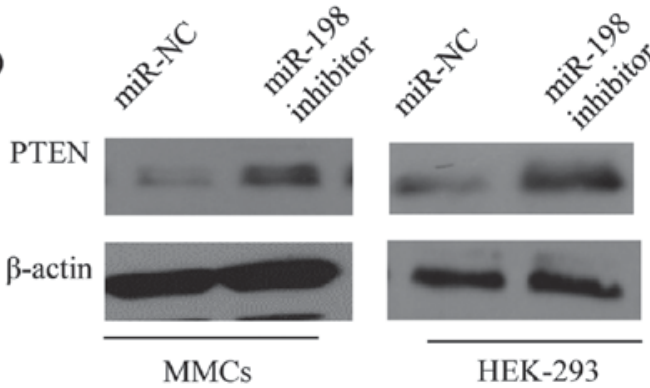

Figure 4. Forced expression of miR-198 inhibits PTEN expression. MMCs and HEK-293 cells were transfected with (A) miR-198 mimic or miR-control, and (B) miR-198 inhibitor or miR-NC. Reverse transcription-quantitative polymerase chain reaction analysis was performed to detect the expression of PTEN. GAPDH served as an internal control. Data are expressed as the mean \pm standard deviation. ${ }^{* *} \mathrm{P}<0.01$. Representative western blot images of PTEN protein expression levels in MMCs and HEK-293 cells were transfected with (C) miR-198 mimic or miR-control, and (D) miR-198 inhibitor or miR-NC. PTEN, phosphatase and tensin homology deleted on chromosome ten; miR, microRNA; NC, negative control.

A

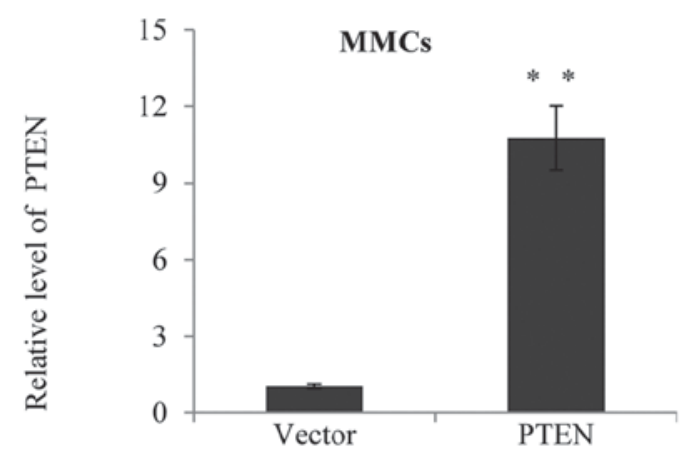

$\mathrm{C}$

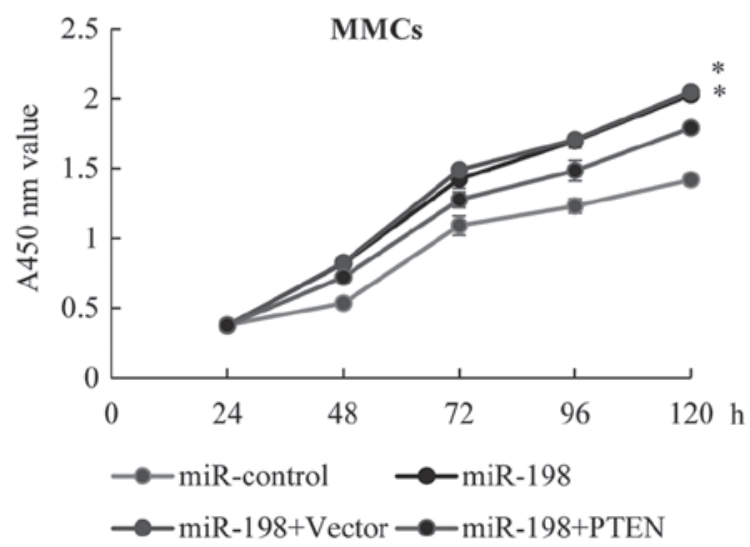

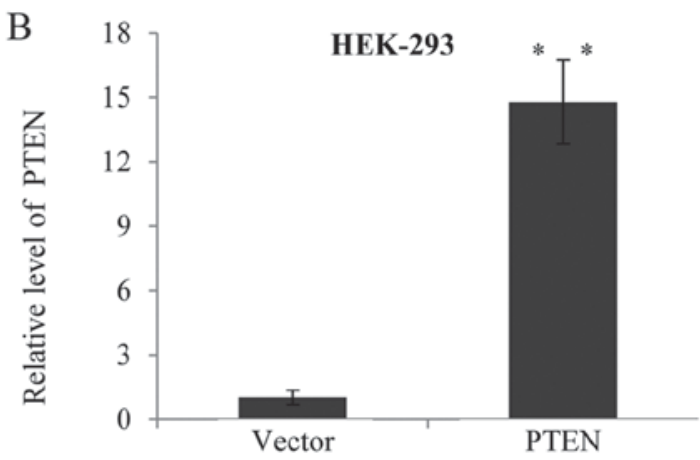

$\mathrm{D}$

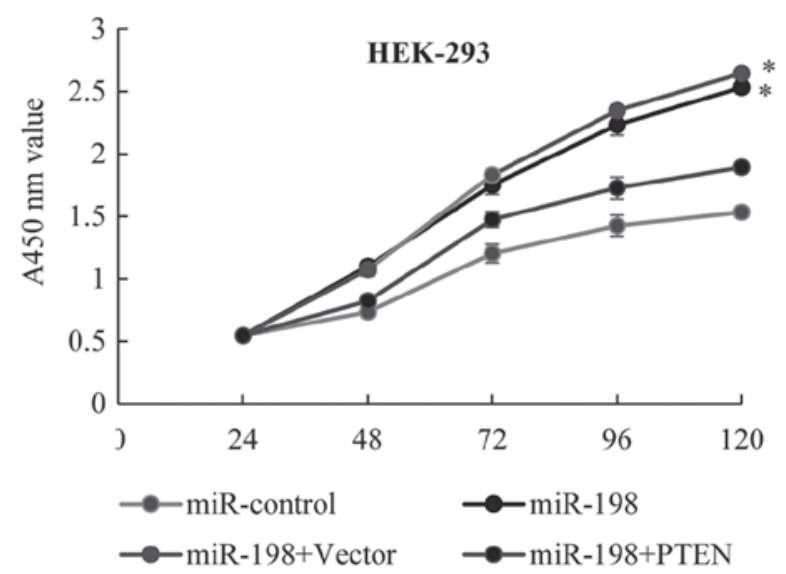

Figure 5. Inhibition of PTEN is involved in the miR-198-mediated MMC proliferation. (A) MMCs and (B) HEK-293 cells were infected with either an empty vector or a lentivirus vector expressing PTEN. Reverse transcription-quantitative polymerase chain reaction analysis was performed to detect the expression of PTEN. Cell Counting kit-assay was performed in (C) MMCs and (D) HEK-293 cells. Data are expressed as the mean \pm standard deviation. ** $<0.01$. PTEN, phosphatase and tensin homology deleted on chromosome ten; miR, microRNA; NC. 
A

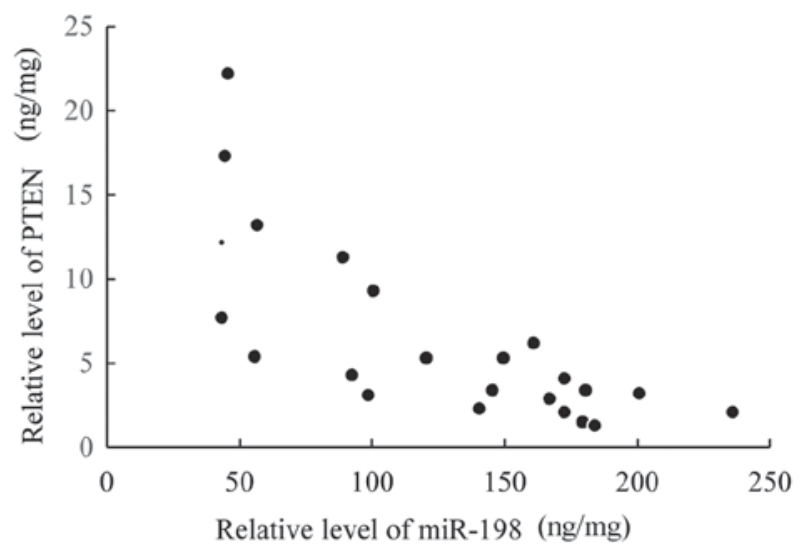

B

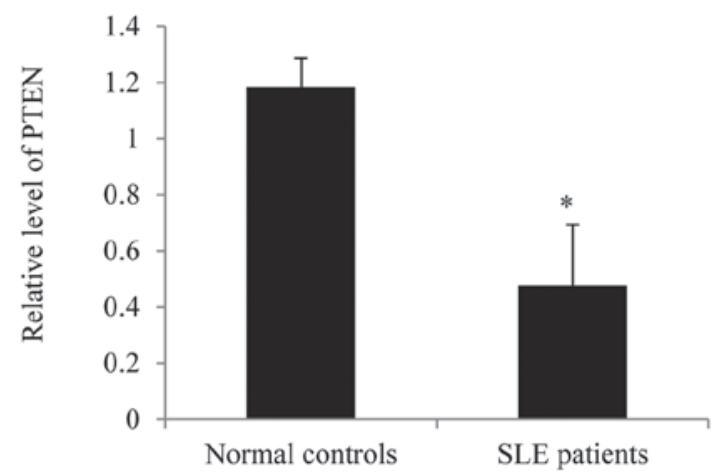

$\mathrm{C}$

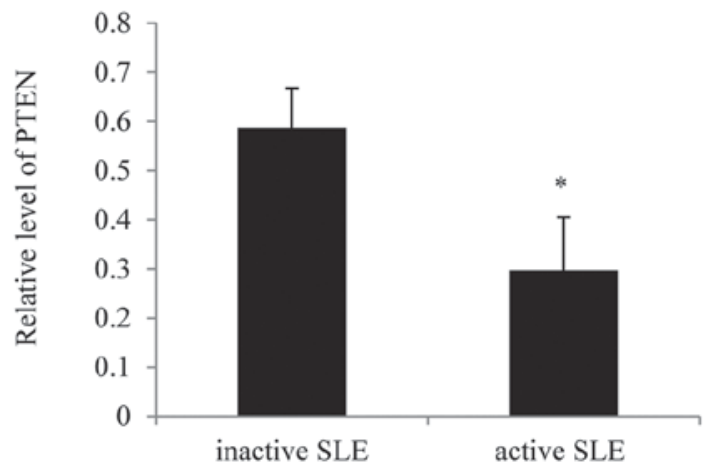

Figure 6. Negative association between miR-198 and PTEN is observed in patients with SLE. (A) Correlation between miR-198 levels and the PTEN levels in the active SLE patients. (B) Expression of PTEN was measured in 52 patients with SLE and 10 normal controls. Data are presented as the mean \pm standard error. (C) Expression of PTEN was measured in 30 patients with active SLE and 22 patients with inactive SLE. Data are presented as the mean \pm standard deviation. ${ }^{*} \mathrm{P}<0.05$. PTEN, phosphatase and tensin homology deleted on chromosome ten; miR, microRNA; SLE, systemic lupus erythematosus

the function of miR-198 on PTEN protein expression. Forced expression of miR-198 mimics significantly reduced the expression of PTEN protein in MMCs and HEK-293 cells (Fig. 4C), whereas the expression of PTEN protein was upregulated after miR-198 was inhibited in the two cell lines (Fig. 4D).

Suppression of PTEN is involved in the miR-198-mediated $M M C$ proliferation. In order to determine the importance of PTEN as a functional target of miR-198, it was hypothesized that forced expression of PTEN could circumvent the inhibitory effects of miR-198 on cell proliferation. A lentiviral vector expressing PTEN was constructed and transfected into the MMCs and HEK-293 cells, and RT-qPCR was used to demonstrate that PTEN expression was restored after PTEN lentiviral infection (Fig. 5A and B). Furthermore, CCK-8 analysis was performed in the above cell lines; compared with the miR-198 groups, forced expression of PTEN rescued the function of miR-198 on cell proliferation (Fig. 5C and D).

Negative association between miR-198 and PTEN is observed in the patients with SLE. To better understand the functionality of miR-198 in SLE progression, the present study assessed the correlation between miR-198 and PTEN levels in the active SLE patients. As presented in Fig. 6A, there was a negative correlation between miR-198 and PTEN. Furthermore, the expression of PTEN was significantly lower in SLE patients compared with normal controls (Fig. 6B). Additionally, the expression of PTEN was lower in patients with active SLE compared with those with inactive SLE (Fig. 6C). These results further support the hypothesis of the present study.

\section{Discussion}

Abnormal miRNA expression is correlated with severity in clinical diseases such as SLE with active LN kidneys $(14,15)$. By interfering with target genes in major intracellular pathways or necrosis and inflammation process, miRNAs serve a critical role in the renal pathophysiology and work as modulators of renal fibrosis (16).

The present study demonstrated an increase in miR-198 expression in LN renal tissues and the MMCs cell line. Furthermore, a novel regulatory mechanism by which PTEN expression was regulated by miR-198 at the post-transcription level was identified, as the mRNA expression level of PTEN remained unchanged while there was a decrease in PTEN protein expression levels. Therefore, miR-198 could accelerate MMC proliferation in vitro while knockdown of miR-198 could suppress MMC proliferation. This result is consistent with previous studies to some extent. For example, miR-198 was identified to be upregulated in the peripheral blood mononuclear cells derived from SLE patients (17). Both glomerular and tubulointerstitial expression of miR-198 is higher in LN patients than controls (18), which is in line with the present study (16). PTEN is a well-known tumor suppressor gene located on chromosome $10(19,20)$. The expression of PTEN is frequently lost or mutated in a variety of human cancers, such as breast, lung and brain cancer $(21,22)$. The present study demonstrated that low PTEN expression level was observed in the renal tissues during LN, and the expression of PTEN was inhibited by miR-148a-3p.

In conclusion, the present study, to the best of our knowledge, is the first to demonstrate that miR-198 is a negative regulator of PTEN, as miR-148a was demonstrated to be previously (23). miR-198 may decrease PTEN expression by binding directly to the 3'UTR, and may serve as a novel and critical factor in the pathogenesis of LN. However, there is a major limitation in the present study in that miR-198 expression levels may change in response to immunosuppressive therapy. Future more studies are required to measure the serial change in the intra-renal expression of miRNAs. These results bring novel insight into understanding the mechanisms about the lupus disease pathogenesis. 


\section{References}

1. Wahren-Herlenius M and Dörner T: Immunopathogenic mechanisms of systemic autoimmune disease. Lancet 382: 819-831, 2013.

2. Cook HT and Botto M: Mechanisms of Disease: The complement system and the pathogenesis of systemic lupus erythematosus. Nat Clin Pract Rheumatol 2: 330-337, 2006.

3. Avihingsanon Y and Hirankarn N: Major lupus organ involvement: Severe lupus nephritis. Lupus 19: 1391-1398, 2010.

4. Maroz N and Segal MS: Lupus nephritis and end-stage kidney disease. Am J Med Sci 346: 319-323, 2013.

5. Borchers AT, Leibushor N, Naguwa SM, Cheema GS Shoenfeld Y and Gershwin ME: Lupus nephritis: A critical review. Autoimmun Rev 12: 174-194, 2012.

6. Zhang B and Farwell MA: microRNAs: A new emerging class of players for disease diagnostics and gene therapy. J Cell Mol Med 12: 3-21, 2008.

7. Taylor DD and Gercel-Taylor C: MicroRNA signatures of tumor-derived exosomes as diagnostic biomarkers of ovarian cancer. Gynecol Oncol 110: 13-21, 2008.

8. Lu LF and Liston A: MicroRNA in the immune system, microRNA as an immune system. Immunology 127: 291-298, 2009.

9. Rusek AM, Abba M, Eljaszewicz A, Moniuszko M, Niklinski J and Allgayer H: MicroRNA modulators of epigenetic regulation, the tumor microenvironment and the immune system in lung cancer. Mol Cancer 14: 34, 2015.

10. Wang G, Tam LS, Li EK, Kwan BC, Chow KM, Luk CC, Li PK and Szeto C: Serum and urinary free microRNA level in patients with systemic lupus erythematosus. Lupus 20: 493-500, 2011

11. Hochberg MC: Updating the American College of Rheumatology revised criteria for the classification of systemic lupus erythematosus. Arthritis Rheum 40: 1725, 1997.

12. Pitashny M, Schwartz N, Qing X, Hojaili B, Aranow C, Mackay M and Putterman C: Urinary lipocalin-2 is associated with renal disease activity in human lupus nephritis. Arthritis Rheum 56: 1894-1903, 2007.

13. Livak KJ and Schmittgen TD: Analysis of relative gene expression data using real-time quantitative PCR and the 2(-Delta Delta C(T)) Method. Methods 25: 402-408, 2001.
14. Lorenzen JM, Haller H and Thum T: MicroRNAs as mediators and therapeutic targets in chronic kidney disease. Nat Rev Nephrol 7: 286-294, 2011.

15. Dai Y, Sui W, Lan H, Yan Q, Huang H and Huang Y: Comprehensive analysis of microRNA expression patterns in renal biopsies of lupus nephritis patients. Rheumatol Int 29: 749-754, 2009.

16. Ichii O, Otsuka-Kanazawa S, Horino T, Kimura J, Nakamura T, Matsumoto M, Toi M and Kon Y: Decreased miR-26a expression correlates with the progression of podocyte injury in autoimmune glomerulonephritis. PLoS One 9: e110383, 2014.

17. Te JL, Dozmorov IM, Guthridge JM, Nguyen KL, Cavett JW, Kelly JA, Bruner GR, Harley JB and Ojwang JO: Identification of unique microRNA signature associated with lupus nephritis. PLoS One 5: e10344, 2010.

18. Lu J, Kwan BC, Lai FM, Tam LS, Li EK, Chow KM, Wang G, Li PK and Szeto CC: Glomerular and tubulointerstitial miR-638, miR-198 and miR-146a expression in lupus nephritis. Nephrology (Carlton) 17: 346-351, 2012.

19. de Assis LV and Isoldi MC: The function, mechanisms, and role of the genes PTEN and TP53 and the effects of asbestos in the development of malignant mesothelioma: A review focused on the genes' molecular mechanisms. Tumour Biol 35: 889-901, 2014.

20. Kitagishi Y and Matsuda S: Redox regulation of tumor suppressor PTEN in cancer and aging (Review). Int J Mol Med 31: 511-515, 2013.

21. Li J, Yen C, Liaw D, Podsypanina K, Bose S, Wang SI, Puc J, Miliaresis C, Rodgers L, McCombie R, et al: PTEN, a putative protein tyrosine phosphatase gene mutated in human brain, breast, and prostate cancer. Science 275: 1943-1947, 1997.

22. Zhang LL, Mu GG, Ding QS, Li YX, Shi YB, Dai JF and Yu HG: Phosphatase and Tensin Homolog (PTEN) represses colon cancer progression through inhibiting paxillin transcription via PI3K/AKT/NF-кB Pathway. J Biol Chem 290: 15018-15029, 2015.

23. Qingjuan L, Xiaojuan F, Wei Z, Chao W, Pengpeng K, Hongbo L, Sanbing Z, Jun H, Min Y and Shuxia L: miR-148a-3p overexpression contributes to glomerular cell proliferation by targeting PTEN in lupus nephritis. Am J Physiol Cell Physiol 310: C470-C478, 2016. 